\title{
Universal depinning transition of domain walls in ultrathin ferromagnets
}

\author{
R. Diaz Pardo, ${ }^{1}$ W. Savero Torres,${ }^{1}$ A. B. Kolton, ${ }^{2}$ S. Bustingorry, ${ }^{2}$ and V. Jeudy ${ }^{1, *}$ \\ ${ }^{1}$ Laboratoire de Physique des Solides, Université Paris-Sud, CNRS, UMR8502, 91405 Orsay, France \\ ${ }^{2}$ CONICET, Centro Atómico Bariloche, 8400 San Carlos de Bariloche, Río Negro, Argentina
}

(Received 26 November 2016; published 30 May 2017)

\begin{abstract}
We present a quantitative and comparative study of magnetic-field-driven domain-wall depinning transition in different ferromagnetic ultrathin films over a wide range of temperature. We reveal a universal scaling function accounting for both drive and thermal effects on the depinning transition, including critical exponents. The consistent description we obtain for both the depinning and subthreshold thermally activated creep motion should shed light on the universal glassy dynamics of thermally fluctuating elastic objects pinned by disordered energy landscapes.
\end{abstract}

DOI: 10.1103/PhysRevB.95.184434

\section{INTRODUCTION}

The depinning transition from a pinned to a sliding state upon increasing a driving force is a nonequilibrium phenomenon observed in extremely diverse systems, ranging from fractures [1,2], charge density waves [3], vortex glasses in superconductors [4,5], ferroelectric [6,7] and ferromagnetic materials [8-11] to wetting [12], reaction fronts [13], and cell migration advancing fronts [14]. At zero temperature, a pinned elastic system presents, upon increasing the driving force $f$, a depinning threshold $f_{d}$ separating the zero velocity state for $f<f_{d}$ from a finite velocity regime for $f>f_{d}$. At finite temperature and below the depinning threshold, the thermal fluctuations result in a so-called "creep" motion over effective pinning barriers. In this dynamical regime, the velocity follows an Arrhenius law $v \sim \exp \left(-\Delta E / k_{B} T\right)$ where $k_{B} T$ is thermal activation (and $k_{B}$ the Boltzmann constant). Close to zero drive $(f \rightarrow 0)$, phenomenological scaling theory $[15,16]$ and functional renormalization group [17] calculations for an elastic line moving in a random pinning disorder predict the effective pinning barriers present a universal power-law variation $\Delta E \sim f^{-\mu}$ with a critical exponent value $\mu=\frac{1}{4}$ which was observed in a real system [18]. More recently, it was shown experimentally that the whole thermally activated dynamical regimes up to the depinning threshold can be universal and controlled by a unique pinning energy barrier function [19].

For the depinning transition, the situation is much less clear since the pinning barriers vanish and their height becomes comparable to thermal activation. Statistical physics approaches predict universal scaling functions of the driving force and temperature for the velocity near depinning [17,20-25]. Above the depinning threshold, it is well established that the velocity displays the critical behavior $v \sim\left(f-f_{d}\right)^{\beta}, \beta$ being the depinning exponent $[3,20,26]$. At finite temperature, the combined effects of drive and thermal noise produce a "thermal rounding" of the velocity-force characteristics. The velocity is usually described by $v \sim T^{\psi}$ at the threshold $\left(f=f_{d}\right)$, with $\psi$ the thermal rounding exponent [20,24,25]. Just above the depinning threshold $\left(f \gtrsim f_{d}\right)$, the combined effects of the drive and thermal noise are conjectured to be caught by $v \sim T^{\psi} g\left[T^{-\psi}\left(f-f_{d}\right)^{\beta}\right]$, where $g$ is a universal scaling function [3,20,22-25]. This

\footnotetext{
*vincent.jeudy@u-psud.fr
}

kind of response, phenomenologically predicted by exploiting the analogy with equilibrium phase transitions, is supported by numerical simulations of a driven elastic string [24,25], the random-field Ising model [21], and charge density waves models [20] but still remains elusive to a rigorous functional renormalization group (FRG) treatment [17]. Experimentally on the other hand, it has been extremely challenging to go beyond critical exponent analysis [1,6-8]. Investigations of the depinning transition is complicated by the thermal rounding of the velocity-force characteristics [24,25] which impedes a straightforward determination of the depinning threshold and consequently a clear distinction between material-dependent and universal behaviors. Therefore, assessing the very existence of a universal scaling function of a reduced force and temperature remains an important open issue.

In this work, we address the question of the universality of the depinning transition in presence of drive and thermal fluctuations, going beyond the determination of critical exponents and we evidence a universal function which captures both the temperature and external drive scaling properties. Strategically, we have chosen to study the motion of domain walls driven by magnetic field in an ultrathin film with perpendicular anisotropy [9,27]. In this system, the magnetic field corresponds to the driving force and the domain walls to elastic one-dimensional lines moving in a two-dimensional medium. The subthreshold creep universal behavior is well understood, so that the nonuniversal material-dependent parameters can be treated consistently. The effective energy barrier [19] $\Delta E \sim\left(H / H_{d}\right)^{-\mu}-1$, with a creep exponent $\mu=\frac{1}{4}[9,17,18]$, describes the whole subthreshold regime up to the depinning threshold. The depinning thresholds as well as the other material-dependent parameters are nonambiguously determined from the methods developed in Ref. [19] and are then used to analyze the depinning transition. At and above threshold, we use the depinning critical exponents $\beta=0.25$ [26] and $\psi=0.15$ [24,25] which are deduced from recent numerical simulations and are compatible with experimental findings [27].

\section{DOMAIN-WALL DYNAMICS}

\section{A. Experimental methods}

In order to test the universal behaviors of depinning transition, the domain-wall dynamics was analyzed for a $\mathrm{Pt} / \mathrm{Co} / \mathrm{Pt}$ 

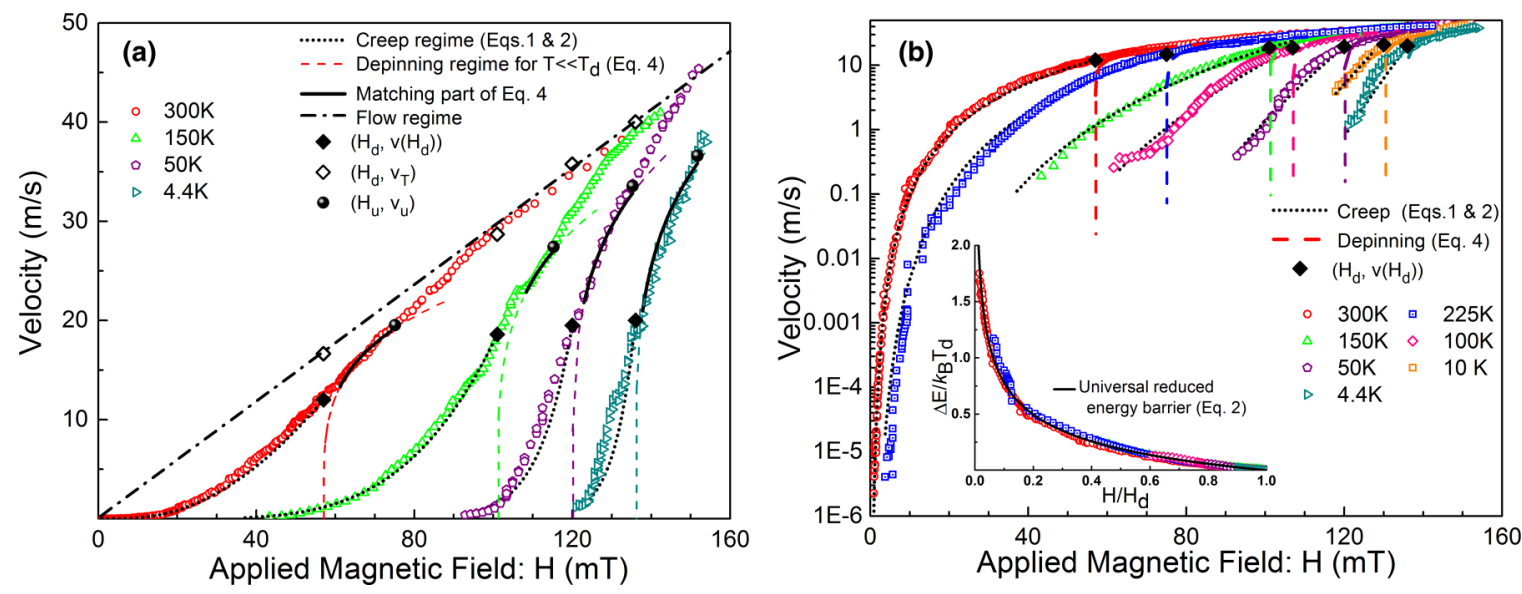

FIG. 1. Domain-wall velocity in Pt/Co/Pt measured for different temperatures and presented in linear (a) and semilog (b) scales to show the high and low drive regimes, respectively. The creep regime $\left[0<H<H_{d}(T)\right]$ is highlighted by the black dotted curves corresponding to fits of Eq. (1) with $\mu=\frac{1}{4}$ and ends for velocities $v\left(H_{d}(T), T\right)$ indicated by black diamond symbols. The depinning regime extends from the depinning threshold $H=H_{d}(T)$ to the universality limit $H_{u}(T)$ (indicated by black solid spheres). The depinning velocities $v_{T}\left(H_{d}(T), T\right)$ deduced from Eq. (3) correspond to empty diamonds. The dashed curves are the predictions of Eq. (4) for a unique value of $x_{0}=v_{T} / v_{H}=0.65$ and match with part of all velocity curves as indicated by black solid segments. The linear flow regime, indicated by a straight dashed-dotted line, is reached for sufficiently high drive (and only for $T>50 \mathrm{~K}$ ). Inset: universal reduced energy barrier as a function of reduced field deduced from velocity curves. The solid line is a plot of Eq. (2).

ultrathin film and then compared to results published in the literature for $\mathrm{Au} / \mathrm{Co} / \mathrm{Au}$ (Ref. [28]) and $\mathrm{CoFeB}$ (Ref. [29]) ultrathin films which present different strengths of pinning disorder.

The $\operatorname{Pt}(3.5 \mathrm{~nm}) / \mathrm{Co}(0.45 \mathrm{~nm}) / \mathrm{Pt}(4.5 \mathrm{~nm})$ ultrathin film was grown by sputtering on an etched $\mathrm{Si} / \mathrm{SiO}_{2}$ substrate [9]. It was placed in an optical He-flow cryostat in order to explore a large range of temperature $(4.4-300 \mathrm{~K})$. For the measurement of domain-wall velocity, we used the bubble expansion method. A set of circular domains were submitted to magnetic field pulses of variable amplitude (between 0 and $160 \mathrm{mT})$ using a small coil $(\sim 100$ turns and $\sim 1 \mathrm{~mm}$ in diameter) placed on the film. Depending on the pulse amplitude, the duration was set from $1 \mu$ s up to $1 \mathrm{~s}$. The motion of domain walls was observed by polar magneto-optical Kerr effect microscopy (PMOKE). The displacement produced by magnetic field pulses was deduced from the difference between images recorded before and after the pulses. The velocity was defined as the ratio between domain-wall displacement and pulse duration. The raw velocity curves were smoothed using a sliding average over five points to facilitate the comparison between predictions and experiments. The obtained velocity curves are shown in Fig. 1. For the lowest temperatures, the low drive limit of the creep regime $\left(H \ll H_{d}\right)$ was beyond experimental access due to the combined large magnetic field amplitude and long pulse duration ( $>60 \mu \mathrm{s}$ ) required to move domain walls. The exploration of the flow regime was limited by multiple nucleation of magnetization reversal occurring during magnetic field pulses.

\section{B. Unified description of the glassy dynamics}

Different dynamic regimes can be identified in the velocityfield curves obtained for the $\mathrm{Pt} / \mathrm{Co} / \mathrm{Pt}$ ultrathin film (see Fig. 1). An inflection point in the velocity-field curve separates the low drive $\left[0<H<H_{d}(T)\right]$ creep regime, where the velocity varies several orders of magnitude over a relatively narrow applied magnetic field range [see Fig. 1(b)], from the depinning regime observed for $\left[H \gtrsim H_{d}(T)\right]$. At sufficient large drive, domain walls follow the flow regime characterized by a linear variation of the velocity [see Fig. 1(a)]. The slope $m=0.28 \pm 0.02 \mathrm{~m} /(\mathrm{s} \mathrm{mT})$ is compatible with results obtained for the asymptotic precessional regime in $\mathrm{Pt} / \mathrm{Co} / \mathrm{Pt}$ ultrathin films of similar thickness [9]. For $\mathrm{Au} / \mathrm{Co} / \mathrm{Au}$ and $\mathrm{Ta} / \mathrm{CoFeB} / \mathrm{MgO}$ films, the velocity curves (see Fig. 2) also present an inflection point corresponding to the depinning threshold $H_{d}(T)$. However, the linear flow regime is not observed at high drive.

In order to discuss those different regimes on a quantitative basis, we propose a unified description of the glassy domainwall dynamics. For the whole creep regime, the domain-wall velocity is described [19] by the following expression:

$$
v(H, T)=v\left(H_{d}, T\right) \exp \left(\frac{-\Delta E}{k_{B} T}\right)
$$

with an effective pinning energy barrier

$$
\Delta E=k_{B} T_{d}\left[\left(\frac{H}{H_{d}}\right)^{-\mu}-1\right],
$$

where $H_{d}$ and $v\left(H_{d}, T\right)$ are the coordinates of the upper boundary of the creep regime $(\Delta E \rightarrow 0)$ and $k_{B} T_{d}$ is a characteristic energy scale measuring the pinning strength. The parameters $v\left(H_{d}(T), T\right), H_{d}(T)$, and $T_{d}(T)$ were determined from the creep motion for each temperature using the method developed in Ref. [19] (see Sec. II C for details and the obtained values). As shown in Fig. 1, the fit of Eqs. (1) and (2) presents a good agreement over the whole creep regime. 

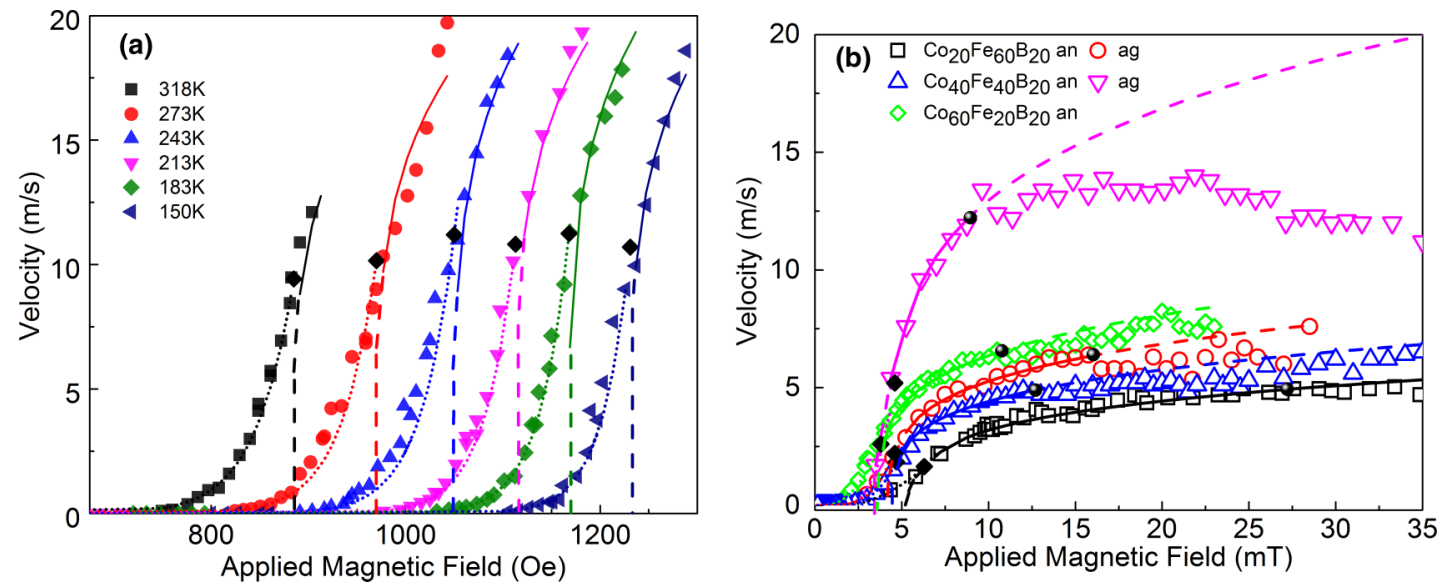

FIG. 2. Domain-wall dynamics observed (a) in $\mathrm{Au} / \mathrm{Co} / \mathrm{Au}$ for different temperatures and (b) in $\mathrm{Ta} / \mathrm{CoFeB} / \mathrm{MgO}$ for different cobalt and iron concentrations and for as-grown (ag) and annealed (an) films. The data are taken from Refs. [28,29], respectively. The diamond symbols represent the upper boundaries of the creep regime $\left[H_{d}(T), v\left(H_{d}, T\right)\right]$. The black solid spheres correspond to the universality limit $H_{u}(T)$. The dotted lines are fits of Eqs. (1) and (2) for the creep regime. The solid and dashed lines correspond to the predictions of Eq. (4) for $x_{0}=0.65$ and is obtained without adjustable parameter.

For the depinning transition, the asymptotic power laws can be written as

$$
v\left(H_{d}, T\right)=v_{T}\left(\frac{T}{T_{d}}\right)^{\psi},
$$

reflecting the variation with temperature of the velocity at the threshold $\left(H=H_{d}\right)$ and

$$
v\left(H, T \ll T_{d}\right)=v_{H}\left(\frac{H-H_{d}}{H_{d}}\right)^{\beta},
$$

corresponding to the variation with magnetic field at zero temperature $\left(T \ll T_{d}\right)$. Here, $v_{T}$ and $v_{H}$ are depinning velocities. Following the more general scaling hypothesis [30], the velocity can be described as a generalized homogeneous function [25,31], which implies the following scaling form:

$$
y=g\left(\frac{x}{x_{0}}\right),
$$

where we have defined the scaled dimensionless variables

$$
x=\left[\left(H-H_{d}\right) / H_{d}\right]^{\beta}\left(T / T_{d}\right)^{-\psi}
$$

and

$$
y=\left(v / v_{T}\right)\left(T / T_{d}\right)^{-\psi},
$$

and where $x_{0}=v_{T} / v_{H}$ is the amplitude ratio [31] of the depinning velocities.

In this model, the function $g$ is expected to be universal within a given class of universality, as it is the case for the critical exponents. The shape of the function $g$ should reflect the two asymptotic behaviors described by Eqs. (3) and (4). For $x \ll x_{0}$, we should have $g\left(x / x_{0}\right) \rightarrow 1$ which corresponds to Eq. (3). On the opposite, for $x \gg x_{0}$, we expect $g\left(x / x_{0}\right) \rightarrow$ $x / x_{0}$ in agreement with the zero-temperature asymptotic limit [Eq. (4) with $v_{H}=v_{T} / x_{0}$.

Therefore, close to the depinning threshold $\left[x \ll x_{0}\right.$, i.e., $\left.H-H_{d} \ll H_{d}\left(T_{d} / T\right)^{-\psi / \beta}\right]$, velocity should depend on temperature while for increasing fields far from the depinning threshold $\left[x \gg x_{0}\right.$, i.e., $\left.H-H_{d} \gg H_{d}\left(T_{d} / T\right)^{-\psi / \beta}\right]$ the velocity should not have an explicit temperature dependence and follow Eq. (4). The precise form of the scaling function $g$ determines the extent of the crossover between these two behaviors, as further discussed in Sec. III E.

Moreover, the parameters $v_{T}, v_{H}, H_{d}$, and $T_{d}$ are nonuniversal material and temperature dependent. As the velocities $v_{T}$ and $v_{H}$ both reflect the dynamics of domain-wall depinning at the same length scale (Larkin length) $[18,27]$ and time scale, their values should be of the same order of magnitude and $x_{0}$ close to 1 . However, since $v_{T}$ and $v_{H}$ act here as purely metric factors in the function $g$ [31], $x_{0}$ is a priori a nonuniversal parameter.

\section{Material- and temperature-dependent parameters}

The material- and temperature-dependent parameters $H_{d}, v\left(H_{d}\right)$, and $T_{d}$ were deduced from Eqs. (1) and (2) describing the creep regime, with the creep exponent $\mu=1 / 4$. For each velocity curve, the following iterative method was used:

(i) Step 1: We assumed the coordinates of the upper boundary of the creep regime $\left[H_{d}, v\left(H_{d}\right)\right]$ to correspond to the inflection point of the velocity characteristics $v(H)$. Indeed, the curvature is predicted to be positive [see Eqs. (1) and (2)] for the creep regime $\left(H<H_{d}\right)$ and to become negative [see Eq. (4)] for the depinning regime $\left(H>H_{d}\right)$.

(ii) Step 2: An estimate of $T_{d}$ is then deduced from a fit of $v(H)$ with Eqs. (1) and (2) over the range $0<H<H_{d}$.

(iii) Step 3: In order to improve the accuracy for the values of $H_{d}$ and $v\left(H_{d}\right)$ a fit of Eqs. (1) and (2) was performed for increasing values of $H$. The upper boundary of the creep regime $\left[H_{d}, v\left(H_{d}\right)\right]$ was defined as the limit above which the fit and the experimental curve start to diverge.

(iv) Steps 2 and 3 were then repeated in order to provide a fine tuning of the values of $H_{d}, v\left(H_{d}\right)$, and $T_{d}$. 
TABLE I. Material- and temperature-dependent parameters. For each material, the thickness [Thick. (nm)] and the temperature of the experiment $[T(\mathrm{~K})]$ is indicated. The fitted parameters are the depinning temperature $\left(T_{d}\right)$, magnetic field $\left(H_{d}\right)$ and the domain-wall depinning velocity $\left(v_{T}\right)$. The coordinates $\left[H_{u}, v\left(H_{u}\right)\right]$ correspond to the upper boundary of the universal behavior. The data depicted for Au/Co/Au and $\mathrm{CoFeB}$ are deduced from results reported in Refs. [28,29], respectively.

\begin{tabular}{|c|c|c|c|c|c|c|c|}
\hline Material & Thick. (nm) & $T(\mathrm{~K})$ & $T_{d}(\mathrm{~K})$ & $H_{d}(\mathrm{mT})$ & $v_{T}(\mathrm{~m} / \mathrm{s})$ & $H_{u}(\mathrm{mT})$ & $v\left(H_{u}\right)(\mathrm{m} / \mathrm{s})$ \\
\hline \multirow[t]{7}{*}{$\mathrm{Pt} / \mathrm{Co} / \mathrm{Pt}$} & \multirow[t]{7}{*}{0.5} & 4.4 & $450(100)$ & $136(3)$ & $40.0(1.0)$ & $151(3)$ & $18.5(1)$ \\
\hline & & 10 & $660(70)$ & $130(1)$ & $39.3(1.0)$ & $138(2)$ & $29(1)$ \\
\hline & & 50 & $2860(150)$ & $120(2)$ & $35.8(1.0)$ & $133(3)$ & $31(2)$ \\
\hline & & 100 & $3090(280)$ & $107(2)$ & $31.3(1.0)$ & $117(2)$ & $27(1)$ \\
\hline & & 150 & $2700(150)$ & 101(1) & $28.7(1.0)$ & $116(2)$ & $27(1)$ \\
\hline & & 225 & $2750(120)$ & $75(1)$ & $21.5(1.0)$ & $96(3)$ & $24(1)$ \\
\hline & & 300 & $2650(20)$ & $57(1)$ & $16.6(1.0)$ & $73(2)$ & $18.5(1)$ \\
\hline \multirow[t]{6}{*}{$\mathrm{Au} / \mathrm{Co} / \mathrm{Au}$} & \multirow[t]{6}{*}{1.0} & 150 & $25800(1000)$ & $123.5(1.0)$ & 23.1(1.0) & $126.5(0.5)$ & $15.8(0.6)$ \\
\hline & & 183 & $28800(1500)$ & $115.0(1.0)$ & $23.9(1.0)$ & $121.5(0.5)$ & $16.7(0.6)$ \\
\hline & & 213 & $29400(1500)$ & $110.0(1.0)$ & $22.8(1.0)$ & $116.0(0.5)$ & $16.9(0.6)$ \\
\hline & & 243 & $29300(1500)$ & $102.5(1.0)$ & $23.0(1.0)$ & $108.5(0.5)$ & $16.5(0.6)$ \\
\hline & & 273 & $29000(1500)$ & $96.5(1.0)$ & $20.5(1.0)$ & $102.0(0.5)$ & $15.5(0.6)$ \\
\hline & & 318 & $28400(1500)$ & $88.0(1.0)$ & $18.5(1.0)$ & $90.5(0.5)$ & $12.2(0.6)$ \\
\hline $\mathrm{Co}_{20} \mathrm{Fe}_{60} \mathrm{~B}_{20}$ an & \multirow[t]{5}{*}{1.0} & \multirow[t]{5}{*}{293} & $1800(100)$ & $6.6(0.2)$ & $2.4(0.5)$ & $27.0(7.0)$ & $4.9(0.4)$ \\
\hline $\mathrm{Co}_{20} \mathrm{Fe}_{60} \mathrm{~B}_{20}$ ag & & & $1800(100)$ & $4.8(0.2)$ & $3.3(0.5)$ & $16.0(3.0)$ & $6.4(0.6)$ \\
\hline $\mathrm{Co}_{40} \mathrm{Fe}_{40} \mathrm{~B}_{20}$ an & & & $1400(100)$ & $5.0(0.2)$ & $2.8(0.5)$ & $12.6(3.0)$ & $4.9(0.6)$ \\
\hline $\mathrm{Co}_{40} \mathrm{Fe}_{40} \mathrm{~B}_{20}$ ag & & & $2000(100)$ & $4.3(0.2)$ & $8.1(0.5)$ & $8.9(2.0)$ & $12.2(2.0)$ \\
\hline $\mathrm{Co}_{60} \mathrm{Fe}_{20} \mathrm{~B}_{20}$ an & & & $2200(100)$ & $3.5(0.5)$ & $3.7(0.5)$ & $10.7(2.0)$ & $6.5(0.5)$ \\
\hline
\end{tabular}

Note that when the flow regime is observed [as in the case of $\mathrm{Pt} / \mathrm{Co} / \mathrm{Pt}$ films, see Fig. $1(\mathrm{a})]$, the coordinates $\left[H_{d}, v\left(H_{d}\right)\right]$ could be also finely adjusted using Eq. (3) and assuming $v_{T}$ to coincide with the velocity of linear flow regime (see the discussion in Sec. III).

The set of temperature-dependent parameters $\left[H_{d}, v\left(H_{d}\right)\right.$, and $T_{d}$ ] are reported in Table I. Equation (1) is then used to plot the reduced energy barrier height $\Delta E / k_{B} T_{d}(T)=$ $\left[T / T_{d}(T)\right] \ln \left[\left(v\left(H_{d}(T), T\right) / v(H, T)\right]\right.$, as a function of the reduced force $H / H_{d}(T)$. As shown in the inset of Fig. 1(b), this transformation collapses all the velocity curves onto a single master curve which reflects the universal behavior of the pinning energy barrier [19]. The good agreement of data with Eq. (2) for $\mu=\frac{1}{4}$ indicates that the whole creep dynamics indeed belongs to the universality class described by the motion of an elastic line with short-range elasticity in a random-bond short-range correlated microscopic pinning potential [19]. The clear discrimination between the universal behavior of the creep energy barrier and the the material- and temperature-dependent pinning properties controlled by the parameters $H_{d}, v\left(H_{d}\right)$, and $T_{d}$ paves the way for a systematic study of domain-wall depinning.

\section{UNIVERSALITY OF THE DEPINNING TRANSITION}

Let us now address the universality of the depinning transition on the basis of Eqs. (3) and (4), using the parameters deduced from the analysis of creep motion, and the critical exponents $\psi=0.15$ and $\beta=0.25$.

\section{A. At the depinning threshold}

At the depinning threshold $\left(H=H_{d}\right)$, Eq. (3) (with $\psi=$ 0.15 ) allows to determine the value of the depinning velocity
$v_{T}$ for different temperatures. As it can be seen in Fig. 1(a), $v_{T}$ presents systematically a good agreement with the velocity corresponding to the linear flow regime. This suggests the depinning velocity $v_{T}$ to correspond to the velocity domain wall would reach in absence of pinning. Moreover, assuming $v_{T}=m H_{d}$, where $m$ is the slope of the flow regime, and inverting Eq. (3), we can estimate the critical exponent $\psi$, directly from the velocity curves. As shown in Fig. 3, $\psi$ is found to be temperature independent and equal to 0.154 \pm 0.006 . The good agreement with previous experimental findings [27] and with numerical predictions [24] is a first signature of the universality of the depinning transition.

\section{B. Upper limit of the depinning transition}

In order to analyze universal behaviors above the depinning threshold, it is particularly important to define a criterion fixing

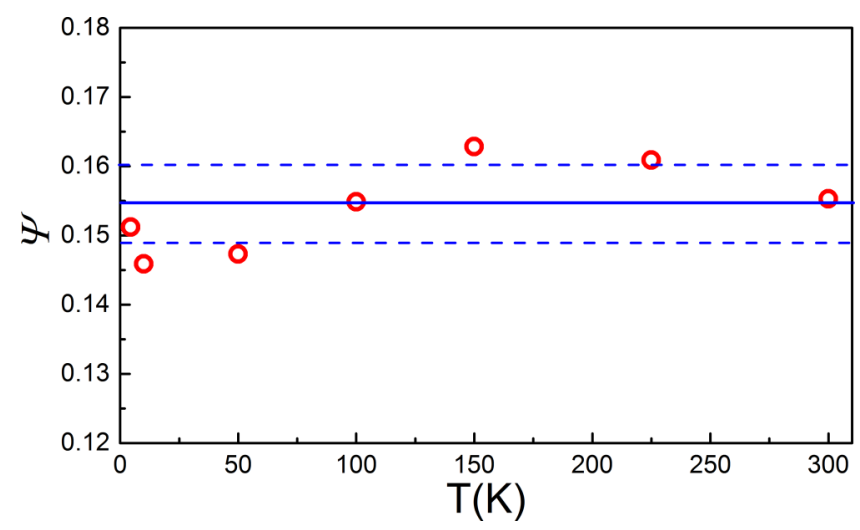

FIG. 3. Depinning critical exponent $\psi$ deduced from experimental curves of Fig. 1 and shown as a function of temperature. 
the upper limit $H_{u}$ of the depinning transition. Above the depinning threshold, since the temperature ratio $T_{d} / T$ is large (see Table I), part of velocity curves should follow the predictions of Eq. (4) with $\beta=0.25$, provided the depinning universality class corresponds to the elastic line model with short-range elasticity in a random-bond short-range correlated microscopic pinning potential, previously shown to be compatible with the observed creep behavior. In order to test this hypothesis, the values of $v_{H}$ were adjusted so that the predictions present the largest matching with the velocity curves [see Fig. 1(a)]. As expected, a good agreement is only obtained over a limited field range. The latter starts slightly above $H_{d}$ $\left(H-H_{d}>1-7 \mathrm{mT}\right.$ for $\left.T=4.4-300 \mathrm{~K}\right)$ due to thermal effect. The onset of the divergence between the experimental curve and the fit of Eq. (4), can define the upper boundary of the depinning regime $\left[H_{u}, v\left(H_{u}\right)\right]$. The obtained values for $\mathrm{Pt} / \mathrm{Co} / \mathrm{Pt}$ as well as for $\mathrm{Au} / \mathrm{Co} / \mathrm{Au}$ and $\mathrm{Ta} / \mathrm{CoFeB} / \mathrm{MgO}$ are reported in Table I. Above $H_{u}(T)$, domain walls should follow an a priori nonuniversal crossover to the linear flow regime observed at larger drive.

\section{Universal depinning velocity ratio}

We now investigate the universality of the whole depinning transition $\left(H_{d}<H<H_{u}\right)$ beyond power laws and critical exponents. Let us first discuss the variations of the ratio $x_{0}=v_{T} / v_{H}$ with the reduced temperature $T_{d} / T$. Surprisingly, $x_{0}$ is found to be temperature independent $\left(x_{0}=0.64 \pm 0.02\right)$ as shown in Fig. 4. As direct consequence, a unique value of $x_{0}$ allows the predictions of Eq. (4) to describe the full set of velocity curves [see Fig. 1(a)]. Moreover, an identical analysis can be performed for other ferromagnetic ultrathin films (see Fig. 2). As shown in Fig. 4, the obtained results present a particularly good agreement for CoFeB [29] $\left(x_{0}=\right.$ $0.64 \pm 0.02)$ and a slightly lower value $x_{0}(=0.62 \pm 0.02)$ for $\mathrm{Au} / \mathrm{Co} / \mathrm{Au}$ [28]. Therefore, as the mean values vary by less than $5 \%$ over wide explored range of reduced temperature $\left(10<T_{d} / T<170\right), x_{0}$ can be reasonably considered as material and temperature independent, which was a priori

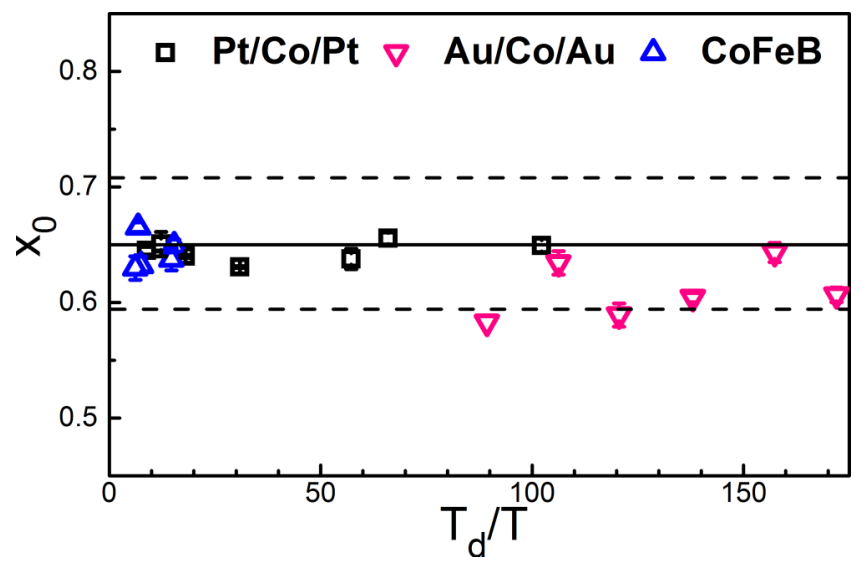

FIG. 4. Universal velocity ratio $x_{0}=v_{T} / v_{H}$ as a function of the reduced temperature $T_{d} / T$ for different ferromagnetic materials. The solid and dashed lines indicate the average value and standard deviation deduced from a fit of the curve in Fig. 5 (see text), respectively. not predicted. This result implies that only three materialand temperature-dependent parameters $\left(v_{T}=x_{0} v_{H}, H_{d}\right.$, and $T_{d}$ ) are sufficient to describe the whole domain-wall glassy dynamics in a universal way.

\section{Universal depinning function}

As discussed in Sec. II B, both magnetic field and temperature effects on domain-wall dynamics should be described by a single function $g$. Figure 5 shows experimental values for the scaled velocity $y$ [see Eq. (7)] as a function of the scaled driving field $x$ [see Eq. (6)]. All the velocity curves collapse onto a single master curve, thus indicating that $x$ and $y$ are appropriate reduced variables to describe the depinning transition and the existence of a universal function $g$. The expected linear asymptotic behavior $g\left(x / x_{0}\right) \rightarrow x / x_{0}$ [derived from Eq. (4)] and corresponding to the low-temperature limit $\left(T_{d} / T \gg 1\right)$ is observed for $x>0.8$. For $x<0.5$, the scaled field $y$ remains almost constant and data extrapolate to $y=1$ for $x \rightarrow 0$ as derived from Eq. (3) (for $H \rightarrow H_{d}$ ). Therefore, the universal $g$ function essentially displays two linear asymptotic behaviors and a narrow crossover region $(0.5<x<0.8)$, which is in qualitative agreement with predictions deduced from numerical simulations which found $x_{0} \sim 1$ [25]. A rather accurate empirical description of data (see the solid lines in Fig. 5) is given by $g(x)=\left[1+\left(\frac{x}{x_{0}}\right)^{n}\right]^{1 / n}$, where $n$ reflects the width of the crossover [32], with a best fit obtained for $x_{0}=0.65 \pm 0.04$ and $n=8.7 \pm 0.4$. This law is also found to be relevant for other magnetic materials. In the inset of Fig. 5, we show the scaling of the velocity data corresponding to $\mathrm{Au} / \mathrm{Co} / \mathrm{Au}$ [28] and $\mathrm{CoFeB}$ [29] using exactly the same function $g$ (i.e., using the same values for $x_{0}$ and $n$ as for

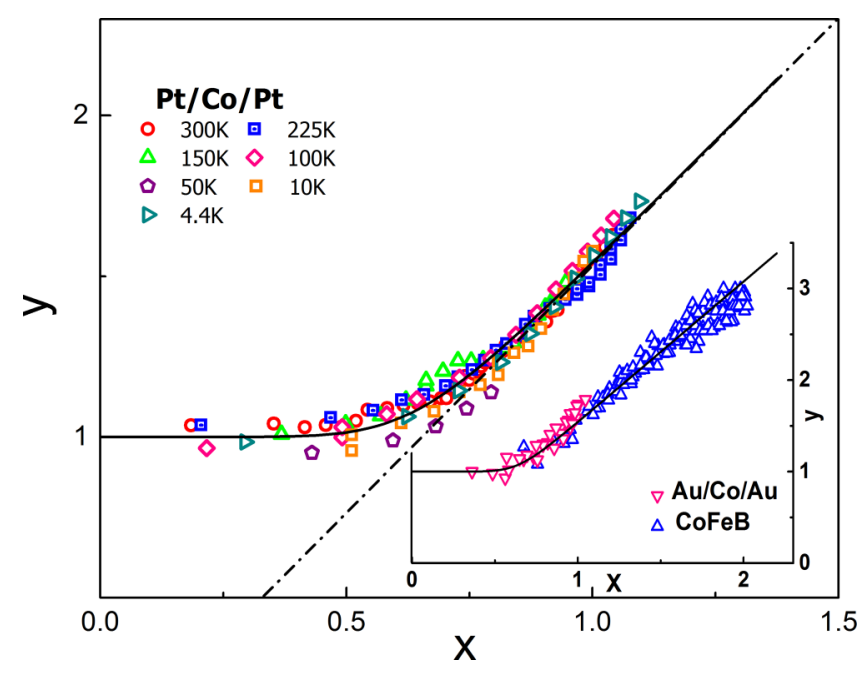

FIG. 5. Universal depinning scaling function obtained using the scaled domain-wall velocity $y$ [see Eq. (7)] as a function of the scaled field $x$ [see Eq. (6)]. The data points only correspond to universal depinning transition $\left(H_{d}<H<H_{u}\right)$. The solid curve (also shown the inset) is an empirical function $g\left(x / x_{0}\right)$ describing the data (see text). The dashed-dotted straight line is the linear asymptotic limit of function $g\left(x / x_{0}\right)$. Inset: scaled domain-wall velocity $y$ as a function of scaled field $x$ for $\mathrm{Au} / \mathrm{Co} / \mathrm{Au}$ [28] and for $\mathrm{CoFeB}$ [29]. 


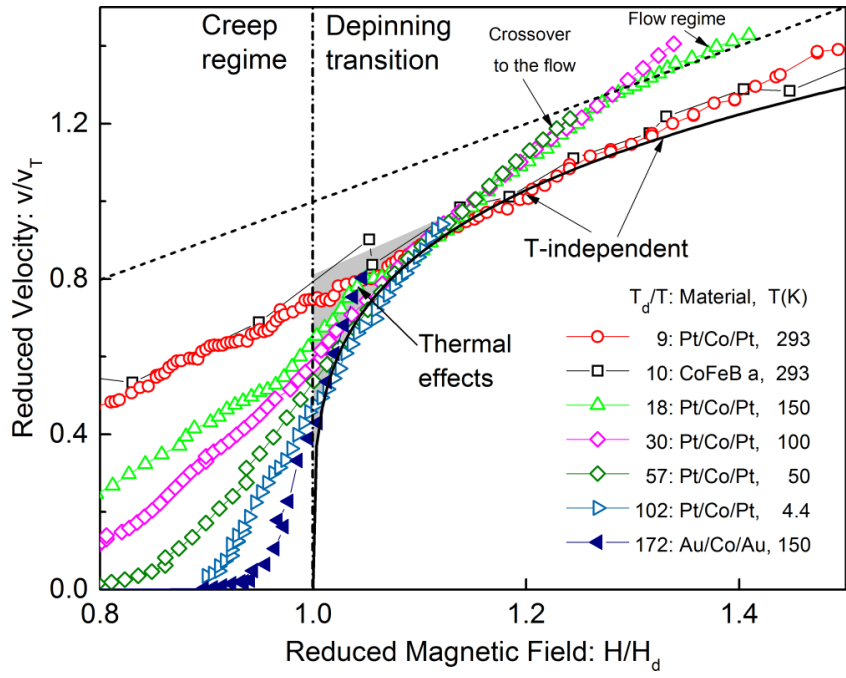

FIG. 6. Domain-wall velocity in reduced coordinates for different reduced temperatures $T_{d} / T$ and materials. The vertical dashed-dotted line corresponds to the depinning threshold which separates the creep regime $\left(H / H_{d}<1\right)$ from the depinning transition $\left(H / H_{d}>1\right)$. The inclined dashed line corresponds to the linear flow regime $\left(v / v_{T}=H / H_{d}\right)$. The solid curve corresponds to the zero-temperature depinning curve, Eq. (4). The gray surface area is the magnetic and velocity ranges over which thermal effects are expected to contribute to the velocity (and which corresponds to the range $x<\tilde{x}=0.8$ of Fig. 5). At higher drive, the reduced velocity becomes independent of temperature as reflected by the merging of velocity curves on the solid curve. Eventually, velocity curves quit the universal depinning transtion at a magnetic field $H_{u}$ which depends on temperature and material and undergo a crossover to the flow regime.

$\mathrm{Pt} / \mathrm{Co} / \mathrm{Pt}$ ), thus bringing further evidence that the depinning transition is well described by a unique universal function.

\section{E. Thermal effects on the depinning velocity}

The shape of the universal depinning function allows to go beyond the usual asymptotic analysis and to address, in particular, the question of the thermal rounding of the depinning transition. Figure 6 presents a comparison of velocity curves in reduced coordinates $\left(v / v_{T}\right.$ vs $\left.H / H_{d}\right)$ for increasing values of the reduced temperature $T_{d} / T$ covering a large range of values (9-172). For the creep regime $\left(H / H_{d}<\right.$ $1)$, the velocity presents a strong variation with temperature associated to the thermal activated nature of the motion [see Eq. (1)]. For the depinning transition, just above the depinning threshold $\left(H / H_{d}=1\right)$, the thermal effects are still important (see the gray area in Fig. 6). The range of magnetic field for which thermal effects are relevant depends on the values of $T_{d} / T$ which reflects the shape of the universal function $g$.
As discussed previously, thermal effects are expected to play a role in the depinning transition only for $x<\tilde{x} \equiv 0.8$ (with $\tilde{y} \equiv$ $\left.\tilde{x} / x_{0}\right)$. Following Eqs. (6) and (7), this corresponds to $H / H_{d}<$ $\tilde{H} / H_{d}=1+\left[\tilde{x}\left(T / T_{d}\right)^{\psi}\right]^{1 / \beta}$ which is the upper magnetic field boundary below which thermal effects are observed in the depinning transition. Concomitantly, a velocity upper limit is given by $\tilde{v}=v_{T} \tilde{x} / x_{0}$. Figure 6 shows indeed that the reduced velocity curves join the predictions of Eq. (4) for a ratio $H / H_{d}$ which decreases as $T_{d} / T$ increases. Moreover, for the explored reduced temperature range, no more thermal effects are observed above $H / H_{d} \approx 1.1$. Above this limit, the reduced velocity displays explicit temperature independence as reflected by the merging of the curves on predictions of Eq. (4).

The crossover between the depinning transition and the flow regime is also highlighted in Fig. 6. The reduced upper limit of the depinning transition $H_{u} / H_{d}$ is found to depend on temperature and to strongly differ between magnetic materials. For $\mathrm{Pt} / \mathrm{Co} / \mathrm{Pt}$, the ratio $H_{u} / H_{d}$ varies between 1.1 and 1.3 over the reduced temperature range $T_{d} / T=9-102$ and it is significantly smaller than for $\mathrm{CoFeB}\left(H_{u} / H_{d}=3-4\right)$. The variation of $H_{u} / H_{d}$ and of $x_{u}(=1.1-2.0)$ suggests that the limit of universality $H_{u}$ is not associated to a universal phenomenon.

\section{CONCLUSION}

In conclusion, the depinning transition of domain walls driven by magnetic field in ultrathin films has been shown to present a universal behavior. The latter is characterized by a universal function of the rescaled field, temperature, and velocity whose shape governs the domain-wall velocity including its asymptotic scaling law behaviors. Moreover, the proposed phenomenological model and a single set of material- and temperature-dependent parameters $\left(H_{d}, T_{d}\right.$, and $v_{T}$ ) are found to allow a self-consistent analysis of both the depinning transition and the subthreshold thermally activated creep regime. Our study thus paves the way for a unified and systematic description of thermal effects in the driven glassy dynamics of disordered elastic systems.

\section{ACKNOWLEDGMENTS}

We wish to thank A. Mougin, A. Thiaville, and the PAREDOM collaboration in Bariloche for fruitful discussions. S.B., J.G., and V.J. acknowledge financial support by the French-Argentina Project ECOS-Sud No. A12E03. This work was also partly supported by the French projects DIM CNano IdF (Region Ile-de-France) and the Labex NanoSaclay, Grant No. ANR-10-LABX-0035. R.D.P. thanks the Mexican council CONACyT for the Ph.D. fellowship No. 449563. S.B. and A.B.K. acknowledge partial support from Project No. PIP11220120100250CO (CONICET).
[1] L. Ponson, Phys. Rev. Lett. 103, 055501 (2009).

[2] L. Laurson, X. Illa, S. Santucci, K. Tore Tallakstad, K. J. Måløy, and M. J. Alava, Nat. Commun. 4, 2927 (2013).

[3] D. S. Fisher, Phys. Rev. B 31, 1396 (1985).
[4] G. Blatter, M. V. Feigel'man, V. B. Geshkenbein, A. I. Larkin, and V. M. Vinokur, Rev. Mod. Phys. 66, 1125 (1994).

[5] P. Le Doussal, Int. J. Mod. Phys. B 24, 3855 (2010). 
[6] J. Y. Jo, S. M. Yang, T. H. Kim, H. N. Lee, J.-G. Yoon, S. Park, Y. Jo, M. H. Jung, and T. W. Noh, Phys. Rev. Lett. 102, 045701 (2009).

[7] B. Li, G. Li, Q. Yin, Z. Zhu, A. Ding, and W. Cao, J. Phys. D: Appl. Phys. 38, 1107 (2005).

[8] S. Zapperi, P. Cizeau, G. Durin, and H. E. Stanley, Phys. Rev. B 58, 6353 (1998).

[9] P. J. Metaxas, J. P. Jamet, A. Mougin, M. Cormier, J. Ferré, V. Baltz, B. Rodmacq, B. Dieny, and R. L. Stamps, Phys. Rev. Lett. 99, 217208 (2007).

[10] S. Bustingorry, A. B. Kolton, and T. Giamarchi, Phys. Rev. B 85, 214416 (2012).

[11] G. Durin, F. Bohn, M. A. Corrêa, R. L. Sommer, P. Le Doussal, and K. J. Wiese, Phys. Rev. Lett. 117, 087201 (2016).

[12] P. L. Doussal, K. J. Wiese, S. Moulinet, and E. Rolley, Europhys. Lett. 87, 56001 (2009).

[13] S. Atis, A. K. Dubey, D. Salin, L. Talon, P. Le Doussal, and K. J. Wiese, Phys. Rev. Lett. 114, 234502 (2015).

[14] O. Chepizhko, C. Giampietro, E. Mastrapasqua, M. Nourazar, M. Ascagni, M. Sugni, U. Fascio, L. Leggio, C. Malinverno, G. Scita, S. Santucci, M. J. Alava, S. Zapperi, and C. A. M. La Porta, Proc. Natl. Acad. Sci. USA 113, 11408 (2016).

[15] M. V. Feigel'man, V. B. Geshkenbein, A. I. Larkin, and V. M. Vinokur, Phys. Rev. Lett. 63, 2303 (1989).

[16] T. Nattermann, Phys. Rev. Lett. 64, 2454 (1990).

[17] P. Chauve, T. Giamarchi, and P. Le Doussal, Phys. Rev. B 62, 6241 (2000).

[18] S. Lemerle, J. Ferré, C. Chappert, V. Mathet, T. Giamarchi, and P. Le Doussal, Phys. Rev. Lett. 80, 849 (1998).
[19] V. Jeudy, A. Mougin, S. Bustingorry, W. Savero Torres, J. Gorchon, A. B. Kolton, A. Lemaître, and J.-P. Jamet, Phys. Rev. Lett. 117, 057201 (2016).

[20] A. A. Middleton, Phys. Rev. B 45, 9465 (1992).

[21] L. Roters, A. Hucht, S. Lübeck, U. Nowak, and K. D. Usadel, Phys. Rev. E 60, 5202 (1999).

[22] P. Le Doussal, K. J. Wiese, and P. Chauve, Phys. Rev. B 66, 174201 (2002).

[23] A. Rosso, A. K. Hartmann, and W. Krauth, Phys. Rev. E 67, 021602 (2003).

[24] S. Bustingorry, A. B. Kolton, and T. Giamarchi, Europhys. Lett. 81, 26005 (2008).

[25] S. Bustingorry, A. B. Kolton, and T. Giamarchi, Phys. Rev. E 85, 021144 (2012).

[26] E. E. Ferrero, S. Bustingorry, and A. B. Kolton, Phys. Rev. E 87, 032122 (2013).

[27] J. Gorchon, S. Bustingorry, J. Ferré, V. Jeudy, A. B. Kolton, and T. Giamarchi, Phys. Rev. Lett. 113, 027205 (2014).

[28] A. Kirilyuk, J. Ferré, V. Grolier, J. P. Jamet, and D. Renard, J. Magn. Magn. Mater. 171, 45 (1997).

[29] C. Burrowes, N. Vernier, J.-P. Adam, L. H. Diez, K. Garcia, I. Barisic, G. Agnus, S. Eimer, J.-V. Kim, T. Devolder, A. Lamperti, R. Mantovan, B. Ockert, E. E. Fullerton, and D. Ravelosona, Appl. Phys. Lett. 103, 182401 (2013).

[30] H. E. Stanley, Introduction to Phase Transitions and Critical Phenomena (Oxford University Press, Oxford, 1971).

[31] V. Privman, P. Hohenberg, and A. Aharony, in Phase Transitions and Critical Phenomena, edited by C. Domb and J. L. Lebowitz (Academic, London, 1991), Vol. 14.

[32] However, let us note that the empirical law reaches an inconsistent zero slope for $n>\beta$ in the limit $H / H_{d} \rightarrow 0$. 